\title{
Computational Solution of a Fractional Integro-Differential Equation
}

\author{
Muhammet Kurulay, ${ }^{1}$ Mehmet Ali Akinlar, ${ }^{2}$ and Ranis Ibragimov ${ }^{3}$ \\ ${ }^{1}$ Department of Mathematics, Yildiz Technical University, 34220 Istanbul, Turkey \\ ${ }^{2}$ Department of Mathematics, Bilecik Seyh Edebali University, 11210 Bilecik, Turkey \\ ${ }^{3}$ Department of Mathematics, University of Texas at Brownsville, Brownsville, TX 78520, USA
}

Correspondence should be addressed to Muhammet Kurulay; mkurulay@yildiz.edu.tr

Received 14 March 2013; Accepted 22 July 2013

Academic Editor: Hassan Eltayeb

Copyright (C) 2013 Muhammet Kurulay et al. This is an open access article distributed under the Creative Commons Attribution License, which permits unrestricted use, distribution, and reproduction in any medium, provided the original work is properly cited.

Although differential transform method (DTM) is a highly efficient technique in the approximate analytical solutions of fractional differential equations, applicability of this method to the system of fractional integro-differential equations in higher dimensions has not been studied in detail in the literature. The major goal of this paper is to investigate the applicability of this method to the system of two-dimensional fractional integral equations, in particular to the two-dimensional fractional integro-Volterra equations. We deal with two different types of systems of fractional integral equations having some initial conditions. Computational results indicate that the results obtained by DTM are quite close to the exact solutions, which proves the power of DTM in the solutions of these sorts of systems of fractional integral equations.

\section{Introduction}

The subject of the present paper is to investigate the applicability of the differential transform method to the systems of the two-dimensional Volterra integro-differential equations of the second kind. To the best of our knowledge, the Volterra-integro differential equations considered in this paper was not studied with any method in the literature. Therefore, solving a new equation with differential transform method is our main purpose in this paper. For this propose, we consider the system of two-dimensional fractional Volterra integro-differential equations in the form of

$$
\begin{gathered}
F_{i}\left(D_{11}^{\alpha} u_{1}(x, t), D_{11}^{\beta} u_{1}(x, t), \ldots, D_{1 m}^{\alpha} u_{m}(x, t),\right. \\
\left.D_{1 m}^{\beta} u_{m}(x, t)\right)
\end{gathered}
$$

$$
\begin{array}{r}
-\lambda_{i} \int_{t_{0}}^{t} \int_{x_{0}}^{x} \sum_{j=0}^{n} v_{i j}(x, t) w_{i j}(y, z) G_{i}\left(D_{11} u_{1}(y, z), \ldots,\right. \\
\left.D_{1 m} u_{m}(y, z)\right) d y d z=f_{i}(x, t), \\
i=1, \ldots, m,
\end{array}
$$

where $\alpha, \beta$ is a parameter describing the fractional derivative. If $0<\alpha, \beta \leq 1$, then the resulting system of fractional integro-differential equations is known as a system of the two-dimensional fractional Volterra integro-differential equations of the second kind.

Fractional calculus basically deals with a generalization of the concept of the ordinary and partial derivative (or differentiation) and integration to arbitrary order including a fractional order. Although the origin of the subject dates back to almost a hundred years ago, recently, this subject has 
been broadly employed in the various fields of engineering and science. Fractional calculus has a broad range of application areas including nonlinear control theory, mathematical biology, plasma physics and fusion, computational fluid mechanics, and images processing.

Integral equations are useful and significant equations in many applications. Problems in which integral equations are encountered include electromagnetic waves, radiative energy transfer, and the oscillation problems. The Volterra integral equations are a special sort of integral equations which are significantly important and useful equations having broad application areas in different branches of science. The Volterra integral equations were first introduced by Volterra and then studied by Lalescu in his thesis. The Volterra integral equations find application in many different areas including sorption kinetics, demography, viscoelastic materials, oscillation of a spring, financial mathematics, stochastic dynamical systems, and mathematical biology.

Fractional differential equations (e.g., see $[1,2]$ ) and fractional integral equations (e.g., see [3]) are a significant research area of recent times. The organization of this paper might be briefly summarized as describing the problem, the DTM method, and applying the method to the problem. Having defined the problem previously, next, we describe the differential transform method shortly.

\section{Differential Transform Method}

The DTM constructs analytical solutions of fractional differential equations in an iterative way in the form of polynomials. DTM is different from the traditional higher order Taylor series techniques which usually demand symbolic computations.

Consider a function of two variables $u(x, y)$, and suppose that it can be represented as a product of two single-variable functions as $u(x, y)=f(x) g(y)$. Now, using the properties of DTM, it is not hard to show that the function $u(x, y)$ can be represented as

$$
\begin{aligned}
u(x, y) & =\sum_{k=0}^{\infty} F_{\alpha}(k)\left(x-x_{0}\right)^{k \alpha} \sum_{h=0}^{\infty} G_{\beta}(h)\left(y-y_{0}\right)^{h \beta} \\
& =\sum_{k=0}^{\infty} \sum_{h=0}^{\infty} U_{\alpha \beta}(k, h)\left(x-x_{0}\right)^{k \alpha}\left(y-y_{0}\right)^{h \beta},
\end{aligned}
$$

where $0<\alpha, \beta \leq 1, U_{\alpha \beta}(k, h)=F_{\alpha}(k) G_{\beta}(h)$ is called the spectrum of $u(x, y)$. The generalized two-dimensional differential transform of the function $u(x, y)$ is given by

$$
\begin{aligned}
U_{\alpha, \beta}(k, h)= & \frac{1}{\Gamma(\alpha k+1) \Gamma(\beta h+1)} \\
& \times\left[\left(D_{* x_{0}}^{\alpha}\right)^{k}\left(D_{* y_{0}}^{\beta}\right)^{h} u(x, y)\right]_{\left(x_{0}, y_{0}\right)},
\end{aligned}
$$

where $\left(D_{x_{0}}^{\alpha}\right)^{k}=D_{x_{0}}^{\alpha} D_{x_{0}}^{\alpha} \cdots D_{x_{0}}^{\alpha}, k$-times. Let us notice that if $u(x, y)=D_{x_{0}}^{\alpha} v(x, y), 0<\alpha \leq 1$, then $U_{\alpha, \beta}(k, h)=(\Gamma(\alpha(k+$
$1)+1) / \Gamma(\alpha k+1)) V_{\alpha, \beta}(k+1, h)$, and the generalized differential transform (3) might be written as

$$
\begin{aligned}
U_{\alpha, \beta}(k, h)= & \frac{1}{\Gamma(\alpha k+1) \Gamma(\beta h+1)} \\
& \times\left[D_{* x_{0}}^{\alpha k}\left(D_{* y_{0}}^{\beta}\right)^{h} u(x, y)\right]_{\left(x_{0}, y_{0}\right)} .
\end{aligned}
$$

If $u(x, y)=D_{x_{0}}^{\gamma} v(x, y), m-1<\gamma \leq m$ and $v(x, y)=$ $f(x) g(y)$, then

$$
U_{\alpha, \beta}(k, h)=\frac{\Gamma(\alpha k+\gamma+1)}{\Gamma(\alpha k+1)} V_{\alpha, \beta}\left(k+\frac{\gamma}{\alpha}, h\right) .
$$

If $u(x, y, t)=D_{* x_{0}}^{\alpha} v(x, y, t), 0<\alpha \leq 1$, then

$$
\begin{gathered}
U_{\alpha, \beta, \gamma}(k, h, m)=\frac{\Gamma(\alpha(k+1)+1)}{\Gamma(\alpha k+1)} V_{\alpha, \beta, m}(k+1, h, m) \\
\text { If } u(x, y)=a(x, y)\left(\partial^{2} v(x, y) / \partial v^{2}(x, y)\right) \text {, then } \\
U(k, h)=\sum_{i=0}^{k} \sum_{j=0}^{h}(k-i+2)(k-i+1) A(i, j) \\
\times U(k-i+2, h-j) .
\end{gathered}
$$

The proofs of some of these properties can be found in [4]. The application of DTM was successfully extended to obtain analytical approximate solutions to some other differential equations of fractional order. Interested reader can take a look at the related papers at [5-7]. As a related work, we can show the work by Bandrowski et al. who studied the numerical solutions of the fractional perturbed Volterra equations in [8]. Next, we illustrate the application of DTM to the systems of fractional integral equations.

\section{Computational Applications}

Example 1. Consider the system of integro-differential equations:

$$
\begin{aligned}
& \frac{\partial^{\alpha} u(x, t)}{\partial t^{\alpha}}+v(x, t) \\
& \quad-\int_{0}^{t} \int_{0}^{x} y \sin z\left(u^{2}(y, z)-v^{2}(y, z)\right) d y d z \\
& =\frac{1}{12}\left(1+2 \cos ^{3} t-3 \cos t\right) x^{4} \\
& \frac{\partial^{\alpha} u(x, t)}{\partial t^{\alpha}}+\frac{\partial^{\alpha} v(x, t)}{\partial t^{\alpha}}+u(x, t) \\
& \quad-\int_{0}^{t} \int_{0}^{x} y \cos z\left(u(y, z)-\frac{\partial v(y, z)}{\partial z}\right) d y d z \\
& =x(2 \cos t-\sin t),
\end{aligned}
$$

with $x, t \in[0,1] u_{1}(x, 0)=x, u_{2}(x, 0)=0, x \in$ $[0,1]$ which has the exact solutions $u_{1}(x, t)=x \cos t$ and 
$u_{2}(x, t)=x \sin t$. Taking the two-dimensional transform of this equation by using the related theorem, we have

$$
\begin{aligned}
& \frac{\Gamma(\alpha(m+1)+1)}{\Gamma(\beta m+1)} U_{\alpha, 1}(m+1, n)-V_{\alpha, 1}(m, n) \\
& -\frac{1}{m n} \sum_{l}^{n-1} \sum_{k}^{m-1} \sum_{s}^{l} \sum_{r}^{k} \sum_{q}^{n-l-1} \sum_{p}^{m-k-1} \frac{\delta_{r, 1} \delta_{s, 0} \delta_{k-r, 0}}{(l-s) !} \sin \left(\frac{(l-s) \pi}{2}\right) \\
& \times\left[U_{\alpha, 1}(p, q) U_{\alpha, 1}(m-k-q-1, n-l-q-1)\right. \\
& \left.-V_{\alpha, 1}(m-k-q-1, n-l-q-1)\right] \\
& =\frac{1}{6} \sum_{l}^{n} \sum_{k}^{m} \sum_{s}^{m-l} \sum_{r}^{m-k} \sum_{q}^{n-l-m} \sum_{p}^{m-k-r} \frac{\delta_{k, 4} \delta_{l, 0} \delta_{r, 0} \delta_{q, 0} \delta_{m-k-r-q, 0}}{s ! q !(n-l-s-q) !} \\
& \times \cos \left(\frac{s \pi}{2}\right) \cos \left(\frac{q \pi}{2}\right) \cos \left(\frac{(n-l-s-q) \pi}{2}\right) \\
& +\frac{1}{12} \delta_{m, 4} \delta_{n, 0}-\frac{1}{4} \sum_{l}^{n} \sum_{k}^{m} \frac{\delta_{k, 4} \delta_{l, 0} \delta_{m-k, 0}}{(n-l) !} \cos \left(\frac{(n-l) \pi}{2}\right), \\
& m=1, \ldots, N-1, n=1, \ldots, N, \\
& \frac{\Gamma(\alpha(m+1)+1)}{\Gamma(\alpha m+1)} U_{\alpha, 1}(m, n+1) \\
& +\frac{\Gamma(\alpha(m+1)+1)}{\Gamma(\alpha m+1)} V_{\alpha, 1}(m, n+1)-U_{\alpha, 1}(m, n) \\
& -\frac{1}{m n} \sum_{l}^{n-1 m-1} \sum_{k}^{l} \sum_{s}^{l} \sum_{r}^{k} \frac{\delta_{r, 1} \delta_{s, 0} \delta_{k-r, 0}}{(l-s) !} \cos \left(\frac{(l-s) \pi}{2}\right) \\
& \times\left[U_{\alpha, 1}(m-k-1, n-l-1)\right. \\
& \left.-(n-1) V_{\alpha, 1}(m-k-1, n-l)\right] \\
& =\sum_{l}^{n} \sum_{k}^{m} \frac{\delta_{k, 1} \delta_{l, 0} \delta_{m-k, 0}}{(n-l) !}\left[2 \cos \left(\frac{(n-l) \pi}{2}\right)-\sin \left(\frac{(n-l) \pi}{2}\right)\right] \text {, } \\
& m=1, \ldots, N, n=1, \ldots, N-1 \text {. }
\end{aligned}
$$

Substituting initial conditions in the equations, we obtain

$$
\begin{gathered}
u_{N}(x, t)=x\left(1+\frac{t^{2 \alpha}}{\Gamma(2 \alpha+1)}+\frac{t^{4 \alpha}}{\Gamma(4 \alpha+1)}+\cdots\right. \\
\left.+\frac{t^{2 N \alpha}}{\Gamma(2 N \alpha+1)}\right), \\
v_{N}(x, t)=x\left(\frac{t^{\alpha}}{\Gamma(\alpha+1)}-\frac{t^{3 \alpha}}{\Gamma(3 \alpha+1)}+\frac{t^{5 \alpha}}{\Gamma(5 \alpha)}+\cdots\right. \\
\left.+\frac{(-1)^{N-1} t^{(2 N-1) \alpha}}{\Gamma((2 N-1) \alpha+1)}\right) .
\end{gathered}
$$

As a special case, if $\alpha=1$, we get the following exact solutions:

$$
u_{1}(x, t)=x \cos t, \quad u_{2}(x, t)=x \sin t
$$

Example 2. In this example, we consider that

$$
\begin{aligned}
& \frac{\partial^{\beta} u(x, t)}{\partial x^{\beta}}-v(x, t)-\int_{0}^{t} \int_{0}^{x} y^{2} u^{2}(y, z) v^{2}(y, z) d y d z \\
& =e^{t}-t e^{-t}-\frac{1}{15} x^{5} t^{3} \\
& \frac{\partial^{\beta} v(x, t)}{\partial x^{\beta}}+u(x, t)-\int_{0}^{t} \int_{0}^{x} z^{2} u(y, z) v(y, z) d y d z \\
& =t e^{t}-\frac{1}{8} x^{2} t^{4},
\end{aligned}
$$

for $x, t \in[0,1]$ and initial conditions $u(x, 0)=0, v(0, t)=$ $t e^{-t}, t \in[0,1]$ which have the exact solutions $u(x, t)=x e^{t}$ and $v(x, t)=t e^{-t}$. Using similar methods as in Example 1, we get

$$
\begin{aligned}
& \frac{\Gamma(\beta(m+1)+1)}{\Gamma(\beta m+1)} U_{1, \beta}(m+1, n)-V_{1, \beta}(m, n) \\
& -\frac{1}{m n} \sum_{l}^{n-1} \sum_{k}^{m-1} \sum_{s}^{n-l-1} \sum_{r}^{m-k-1} \sum_{j}^{s} \sum_{i}^{s} \sum_{p}^{n-l-s-1} \sum_{q}^{m-k-r-1} \\
& \times\left[\delta_{k, 2} \delta_{l, 0} U_{1, \beta}(i, j) U_{1, \beta}(r-i, s-j) V_{1, \beta}(p, q)\right. \\
& \left.\quad V_{1, \beta}(m-k-r-q-1, n-l-s-q-1)\right] \\
& -\frac{1}{n !} \delta_{m, 0}-\frac{(-1)^{n-1}}{(n-1) !}-\frac{1}{15} \delta_{m, 5} \delta_{n, 3}, \\
& m=1, \ldots, N-1, n=1, \ldots, N
\end{aligned}
$$

and for the second equation,

$$
\begin{aligned}
& \frac{\Gamma(\beta(m+1)+1)}{\Gamma(\beta m+1)} V_{1, \beta}(m+1, n)+U_{1, \beta}(m, n) \\
& -\frac{1}{m n} \sum_{l}^{n-1} \sum_{k}^{m-1} \sum_{s}^{n-l-1} \sum_{r}^{m-k-1} \\
& \times\left[\delta_{k, 0} \delta_{l, 2} U_{1, \beta}(i, j) U_{1, \beta}(r, s) V_{1, \beta}(p, q)\right. \\
& \left.\quad V_{1, \beta}(m-k-r-1, n-l-s-1)\right] \\
& =\frac{1}{n !} \delta_{m, 1}-\frac{1}{8} \delta_{m, 2} \delta_{n, 4}, \quad m=1, \ldots, N-1, n=1, \ldots, N
\end{aligned}
$$

From the initial conditions, we get

$$
\begin{gathered}
U(0, n)=0, \quad n=0,1,2, \ldots, N, \\
V(0,0)=0, \quad V(0, n)=\frac{(-1)^{n-1}}{(n-1) !}, \quad n=1,2, \ldots, N .
\end{gathered}
$$

Substituting $x=0$ in the second equation and using the second condition, we obtain

$$
\frac{\partial u_{1}}{\partial x}(0, t)=e^{t}
$$


Substituting initial conditions into the equations, we obtain

$$
\begin{aligned}
u_{N}(x, t)= & x\left(1+\frac{t}{\Gamma(1+1)}+\frac{t^{2}}{\Gamma(2+1)}+\cdots\right. \\
& \left.+\frac{t^{N}}{\Gamma(N+1)}\right), \\
v_{N}(x, t)= & \frac{t}{\Gamma(+1)}-\frac{t^{2}}{\Gamma(2+1)} \\
& +\frac{t^{3 \beta}}{\Gamma(3+1)}+\cdots+\frac{(-1)^{N-1} t^{N}}{\Gamma(N)},
\end{aligned}
$$

where $u_{N}$ and $v_{N}$ are approximate solutions of $u$ and $v$.

For the special case $(\beta=1)$, we can reproduce the series solution of Example 2 and the solution in a closed form $u(x, t)=x e^{t}$ and $v(x, t)=t e^{-t}$.

\section{Conclusions}

The application of the differential transform method has been successfully employed to obtain the approximate analytical solutions for two classes of systems of the two-dimensional fractional Volterra integro-differential equations of the second kind. The method was used in a direct way without using linearization, perturbation, or restrictive assumptions. When $\alpha=1$ and $\beta=1$, we conclude that our approximate solutions are in good agreement with the exact values.

\section{References}

[1] I. Podlubny, Fractional Differential Equations, Academic Press, San Diego, Calif, USA, 1999.

[2] M. Kurulay and M. Bayram, "Some properties of the MittagLeffler functions and their relation with the Wright functions," Advances in Difference Equations, vol. 2012, p. 181, 2012.

[3] A. Kadem and A. Kilicman, "The approximate solution of fractional fredholm integro differential equations by variational iteration and homotopy perturbation methods," Abstract and Applied Analysis, vol. 2012, Article ID 486193, 10 pages, 2012.

[4] S. Momani and Z. Odibat, "A novel method for nonlinear fractional partial differential equations: combination of DTM and generalized Taylor's formula," Journal of Computational and Applied Mathematics, vol. 220, no. 1-2, pp. 85-95, 2008.

[5] A. Secer, M. A. Akinlar, and A. Cevikel, "Efficient solutions of systems of fractional PDEs by differential transform method," Advances in Difference Equations, vol. 2012, p. 188, 2012.

[6] M. Kurulay and M. Bayram, "Approximate analytical solution for the fractional modified $\mathrm{KdV}$ by differential transform method," Communications in Nonlinear Science and Numerical Simulation, vol. 15, no. 7, pp. 1777-1782, 2010.

[7] M. Kurulay, B. A. Ibrahimoğlu, and M. Bayram, "Solving a system of nonlinear fractional partial differential equations using three dimensional differential transform method," International Journal of Physical Sciences, vol. 5, no. 6, pp. 906-912, 2010.

[8] B. Bandrowski, A. Karczewska, and P. Rozmej, "Numerical solutions to fractional perturbed volterra equations," Abstract and Applied Analysis, vol. 2012, Article ID 529602, 19 pages, 2012. 


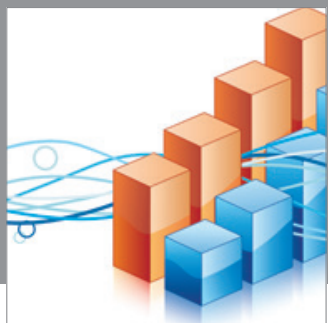

Advances in

Operations Research

mansans

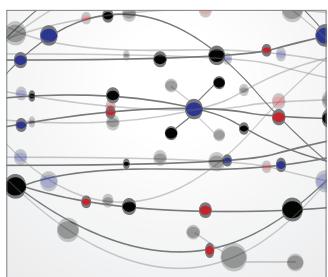

The Scientific World Journal
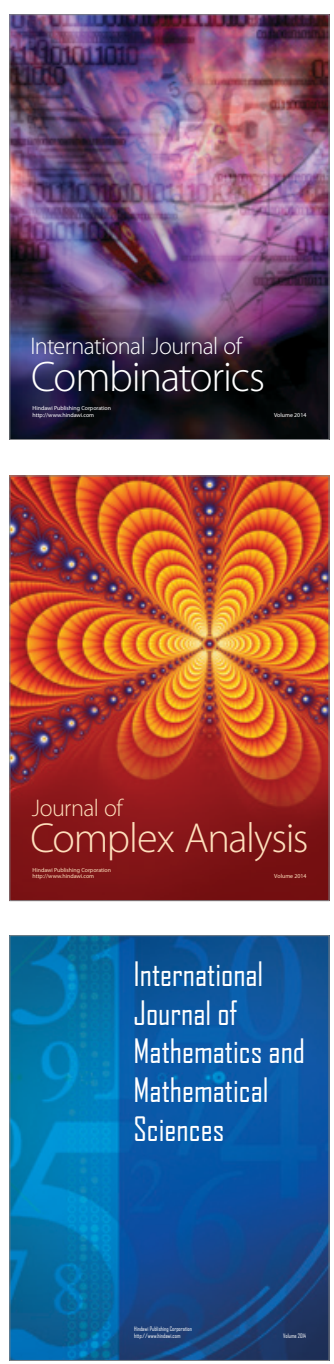
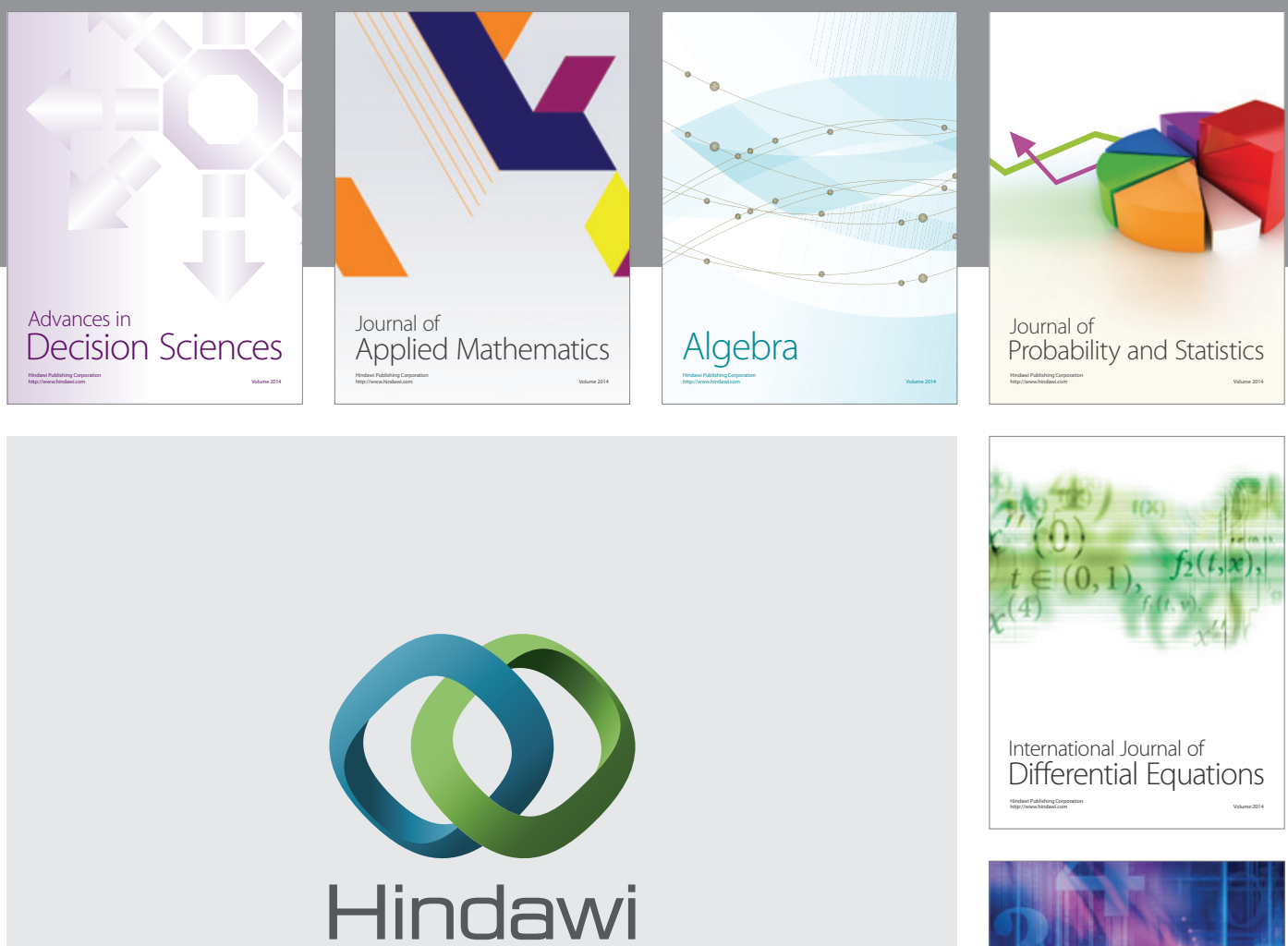

Submit your manuscripts at http://www.hindawi.com
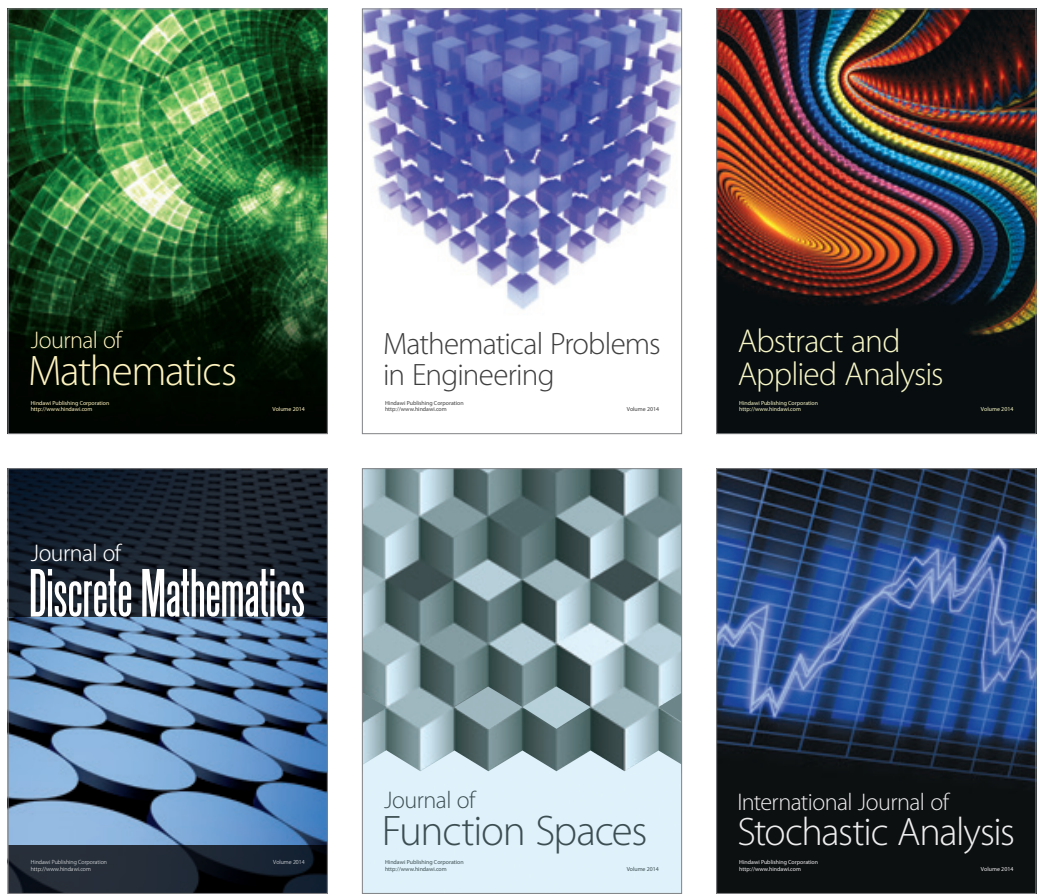

Journal of

Function Spaces

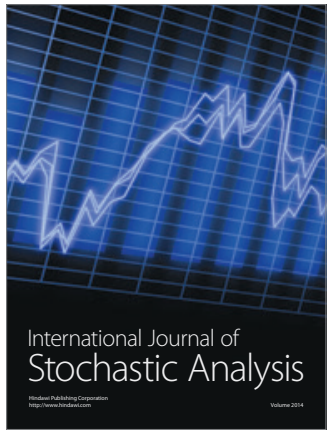

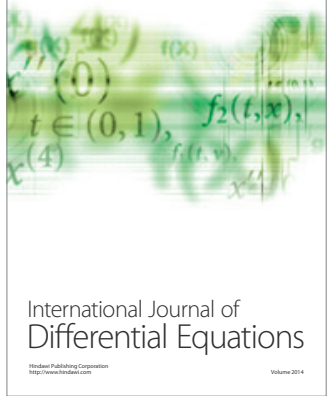
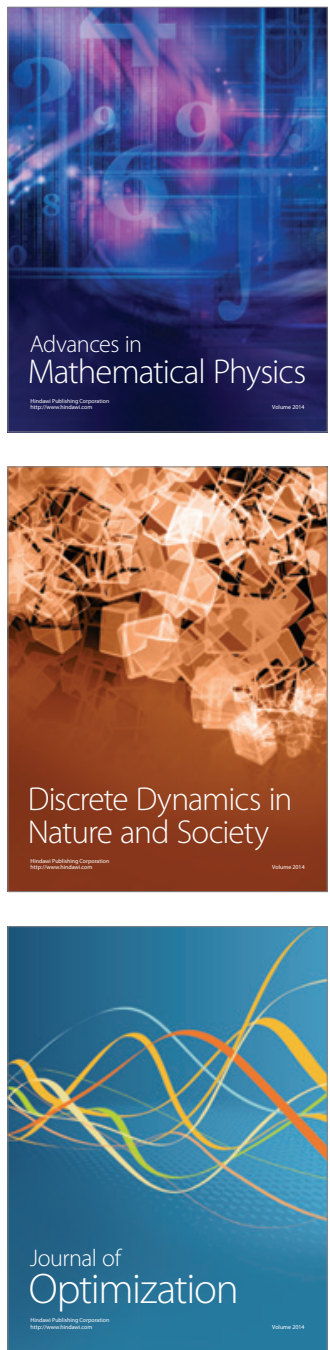